\title{
GROWTH AT INFINITY OF A POLYNOMIAL WITH A COMPACT ZERO SET
}

\author{
JANUSZ GWOŹDZIEWICZ \\ Kielce University of Technology \\ Al. Tysiaclecia Państwa Polskiego 7, 25-314 Kielce, Poland \\ E-mail: matjg@tu.kielce.pl
}

1. Introduction. In the article we give the explicit bound for the growth at infinity of a polynomial with a compact set of zeros. Our aim is to prove the following theorem:

TheOREM 1. Let $F \in \mathbf{R}\left[X_{1}, \ldots, X_{n}\right]$ be a polynomial of degree $d>2$ such that the set $F^{-1}(0)$ is compact. Then there exist constants $c, R>0$ such that

$$
|F(x)| \geq c|x|^{d-(d-1)^{n}} \quad \text { for all } \quad|x|>R .
$$

Recall that we have a similar estimation in the complex case. Consider a polynomial map $H: \mathbf{C}^{n} \rightarrow \mathbf{C}^{n}$ of degree $d$ such that $H^{-1}(0)$ is finite. Then, by Kollár's theorem, $|H(z)| \geq$ const. $|z|^{d-d^{n}}$ for $|z| \gg 1$ (see $[\mathrm{Ko}]$ ). Our theorem is a real counterpart of this inequality.

2. Two lemmas. The following lemmas will be used in the proof of the main theorem.

Lemma 1. Let $G: \mathbf{R}^{n} \rightarrow \mathbf{R}$ be a polynomial of positive degree $d$. Then there exists a linear automorphism $L: \mathbf{R}^{n} \rightarrow \mathbf{R}^{n}$ such that the polynomial $F=G \circ L$ satisfies the following conditions:

(i) All partial derivatives of $F$ are of degree $d-1$.

(ii) The sets $\Gamma_{i}=\left\{x \in \mathbf{R}^{n} \mid \partial F / \partial X_{1}(x)=\ldots=\partial F / \partial X_{i-1}(x)=\partial F / \partial X_{i+1}(x)=\right.$ $\left.\ldots=\partial F / \partial X_{n}(x)=0, \partial F / \partial X_{i}(x) \neq 0\right\}(1 \leq i \leq n)$ are one-dimensional submanifolds of $\mathbf{R}^{n}$ whenever they are not empty,

(iii) For every $x \in \Gamma_{i}(1 \leq i \leq n)$ the differentials $\mathrm{d}_{x}\left(\partial F / \partial X_{1}\right), \ldots, \mathrm{d}_{x}\left(\partial F / \partial X_{i-1}\right)$, $\mathrm{d}_{x}\left(\partial F / \partial X_{i+1}\right), \ldots, \mathrm{d}_{x}\left(\partial F / \partial X_{n}\right)$ are linearly independent.

Research supported by KBN grant 8 P03A 07908.

1991 Mathematics Subject Classification: 14P10.

Received by the editors: December 3, 1996; in the revised form: June 2, 1998.

The paper is in final form and no version of it will be published elsewhere. 
Proof. Let $G L(n)$ be the set of linear automorphisms of $\mathbf{R}^{n}$. We claim that

$$
\left\{L \in G L(n) \mid \operatorname{deg} \frac{\partial G \circ L}{\partial X_{1}}=\ldots=\operatorname{deg} \frac{\partial G \circ L}{\partial X_{n}}=d-1\right\}
$$

is a dense subset of $G L(n)$. Let $G_{d}\left(X_{1}, \ldots, X_{n}\right)$ be the leading form of the polynomial $G$ that is the homogeneous polynomial of degree $d$ for which $\operatorname{deg}\left(G-G_{d}\right)<d$. Consider a substitution $G_{d} \circ L$ where $L\left(X_{1}, \ldots, X_{n}\right)=\left(\sum_{i=1}^{n} l_{1}^{i} X_{i}, \ldots, \sum_{i=1}^{n} l_{n}^{i} X_{i}\right)$. We have $\left(G_{d} \circ L\right)\left(X_{1}, \ldots, X_{n}\right)=G_{d}\left(\sum_{i=1}^{n} l_{1}^{i} X_{i}, \ldots, \sum_{i=1}^{n} l_{n}^{i} X_{i}\right)=G_{d}\left(l_{1}^{1}, \ldots, l_{n}^{1}\right) X_{1}^{d}+\ldots$ $\ldots+G_{d}\left(l_{1}^{n}, \ldots, l_{n}^{n}\right) X_{n}^{d}+$ other monomials. If $G_{d}\left(l_{1}^{1}, \ldots, l_{n}^{1}\right) \neq 0, \ldots, G_{d}\left(l_{1}^{n}, \ldots, l_{n}^{n}\right) \neq 0$, then all partial derivatives of $G \circ L$ are of degree $d-1$. Since the set $\{L \in G L(n)$ $\left.G_{d}\left(l_{1}^{1}, \ldots, l_{n}^{1}\right) \neq 0, \ldots, G_{d}\left(l_{1}^{n}, \ldots, l_{n}^{n}\right) \neq 0\right\}$ is a complement of a proper algebraic set, it is open and dense in $G L(n)$. This proves the claim.

For any $x=\left(x_{1}, \ldots, x_{n}\right)$ from $\mathbf{R}^{n} \backslash\{0\}$ we denote by $[x]$ the corresponding point $\left[x_{1}, \ldots, x_{n}\right]$ of the projective space $\mathbf{R} P^{n-1}$. Consider the map

$$
[\operatorname{grad} G]: \mathbf{R}^{n} \backslash(\operatorname{grad} G)^{-1}(0) \rightarrow \mathbf{R} P^{n-1} .
$$

From the semialgebraic version of Sard's lemma (see [BR], page 82) it follows that the set of regular values of this map contains an open subset $U \subset \mathbf{R} P^{n-1}$. The set $V=$ $\left\{\left(v^{1}, \ldots, v^{n}\right) \in \mathbf{R}^{n} \times \ldots \times \mathbf{R}^{n} \mid \operatorname{det}\left(v_{i}^{j}\right) \neq 0,\left[v^{i}\right] \in U\right.$ for $\left.i=1, \ldots, n\right\}$ is an open subset of $\mathbf{R}^{n} \times \ldots \times \mathbf{R}^{n}$. Each $n$-tuple $v=\left(v^{1}, \ldots, v^{n}\right)$ from this set yields a linear automorphism $A_{v}: \mathbf{R}^{n} \rightarrow \mathbf{R}^{n}, A_{v}(x)=\left(\left\langle v^{1}, x\right\rangle, \ldots,\left\langle v^{n}, x\right\rangle\right)$. Hence the set $\left\{A_{v} \in G L(n) \mid v \in V\right\}$ is open in $G L(n)$. Since $G L(n) \ni A \rightarrow A^{-1} \in G L(n)$ is an open map, $\left\{A_{v}^{-1} \in G L(n) \mid v \in V\right\}$ is also an open subset of $G L(n)$. Thus, there exists $v=\left(v^{1}, \ldots, v^{n}\right) \in V$ such that the automorphism $L=A_{v}^{-1}$ satisfies (i).

Let us define the polynomial $F=G \circ L$. Since $G=F \circ A_{v}, \operatorname{grad} G=A_{v}^{T} \circ \operatorname{grad} F \circ A_{v}$, where $A_{v}^{T}$ is the adjoint of $A_{v}$. From this equation it follows that for any $w \in \mathbf{R}^{n} \backslash\{0\}$, $[w]$ is a regular value of $[\operatorname{grad} F]$ if and only if $\left[A_{v}^{T}(w)\right]$ is a regular value of $[\operatorname{grad} G]$. Let $e^{1}=(1, \ldots, 0), \ldots, e^{n}=(0, \ldots, 1)$ form the standard basis of $\mathbf{R}^{n}$. Since $A_{v}^{T}\left(e^{i}\right)=v^{i}$ for $i=1, \ldots, n$, we conclude that $\left[e^{1}\right], \ldots,\left[e^{n}\right]$ are regular values of $[\operatorname{grad} F]$. Applying the implicit function theorem to the map $[\operatorname{grad} F]$ we see that each of the sets $\Gamma_{i}=$ $[\operatorname{grad} F]^{-1}\left(\left[e^{i}\right]\right)(1 \leq i \leq n)$ is either a one-dimensional submanifold of $\mathbf{R}^{n}$ or is empty. This proves (ii).

We prove the third part of the lemma for $\Gamma_{n}$. To simplify the notation we write $\partial_{i} F$ for $\partial F / \partial X_{i}$. Because $\left[e^{n}\right]$ is a regular value of $[\operatorname{grad} F], 0 \in \mathbf{R}^{n-1}$ is a regular value of the map $\psi: \mathbf{R}^{n} \backslash\left(\partial_{n} F\right)^{-1}(0) \rightarrow \mathbf{R}^{n-1}, \psi=\left(\partial_{1} F / \partial_{n} F, \ldots, \partial_{n-1} F / \partial_{n} F\right)$ that is, the map $[\operatorname{grad} F]$ written in the coordinates $\left\{\left[x_{1}, \ldots, x_{n}\right] \in \mathbf{R} P^{n-1} \mid x_{n} \neq 0\right\} \ni$ $\left[x_{1}, \ldots, x_{n}\right] \rightarrow\left(x_{1} / x_{n}, \ldots, x_{n-1} / x_{n}\right) \in \mathbf{R}^{n-1}$. Therefore, for every $x \in \Gamma_{n}$ the differentials $\mathrm{d}_{x}\left(\partial_{1} F / \partial_{n} F\right), \ldots, \mathrm{d}_{x}\left(\partial_{n-1} F / \partial_{n} F\right)$ are linearly independent. On the other hand, for $x \in \Gamma_{n}$ and $i=1, \ldots, n-1$ we have $\mathrm{d}_{x}\left(\partial_{i} F / \partial_{n} F\right)=\left(1 / \partial_{n} F\right) \mathrm{d}_{x}\left(\partial_{i} F\right)$, therefore the differentials $\mathrm{d}_{x}\left(\partial_{1} F\right), \ldots, \mathrm{d}_{x}\left(\partial_{n-1} F\right)$ are also linearly independent. The proof for $\Gamma_{i}$, $i \neq n$ is similar.

Further, we denote by $|x|$ the supremum norm $|x|=\max \left\{\left|x_{1}\right|, \ldots,\left|x_{n}\right|\right\}$ for $x=$ $\left(x_{1}, \ldots, x_{n}\right)$. We will also use the following convention: Using notation $|x| \gg 1$ we mean that the corresponding condition is satisfied for $|x|>R$, where $R$ is sufficiently large.

LEMma 2. Let $F \in \mathbf{R}\left[X_{1}, \ldots, X_{n}\right]$ be a polynomial with a compact set of zeros and let $K=\left\{x \in \mathbf{R}^{n}\left|\forall y \in \mathbf{R}^{n} \quad\right| y|=| x|\Rightarrow| F(y)|\geq| F(x) \mid\right\}$. If $A \subset K$ is an unbounded semialgebraic set, then the following conditions are equivalent: 
(i) $|F(x)| \geq c|x|^{\alpha} \quad$ for $|x| \gg 1$,

(ii) $|F(x)| \geq c|x|^{\alpha} \quad$ for $|x| \gg 1, x \in A$.

Proof. The implication (i) $\Rightarrow$ (ii) is obvious. Assume that (ii) is true. Since $\{|x| \mid x \in A\}$ is an unbounded semialgebraic subset of $\mathbf{R}_{+}$, there exists a constant $R>0$ such that $(R, \infty) \subset\{|x| \mid x \in A\}$. By (ii) we can choose $R$ sufficiently large so that $|F(x)| \geq c|x|^{\alpha}$ for $|x| \geq R, x \in A$. Let $y \in \mathbf{R}^{n}$ be an arbitrary point with $|y|>R$. Then there exists $x \in A$ such that $|x|=|y|$. By (ii) and the definition of $K$ we get $|F(y)| \geq|F(x)| \geq c|x|^{\alpha}=c|y|^{\alpha}$ which ends the proof.

3. Proof of Theorem 1. The proof proceeds by induction on the number of variables. For polynomials in one variable the theorem is obvious. Assume that the theorem holds for polynomials in $n-1$ variables. We shall check that it is true for polynomials in $n$ variables.

We shall perform some reductions:

If the theorem is true for a polynomial $F$, then it holds also for $F \circ L$, where $L: \mathbf{R}^{n} \rightarrow \mathbf{R}^{n}$ is a linear automorphism. Therefore, we can assume that $F$ satisfies the conditions (i), (ii) and (iii) of Lemma 1.

The set $F^{-1}(0)$ is bounded. Hence $F(x) \neq 0$ for all $|x|>R$, where $R$ is sufficiently large. Since for $n \geq 2$ the set $\left\{x \in \mathbf{R}^{n}|| x \mid>R\right\}$ is connected, a sign of $F$ restricted to $\left\{x \in \mathbf{R}^{n}|| x \mid>R\right\}$ does not change. Without loss of generality we can assume that $F(x)>0$ for $|x|>R$.

Let

$$
K=\left\{x \in \mathbf{R}^{n}\left|\forall y \in \mathbf{R}^{n} \quad\right| y|=| x|\Rightarrow| F(y)|\geq| F(x) \mid\right\} .
$$

First, we prove the theorem under the additional assumption that $K \cap(\operatorname{grad} F)^{-1}(0)$ is unbounded. Let $A$ be an unbounded connected component of this set. Since grad $F(x)=0$ for $x \in A$, we conclude that $\left.F\right|_{A}=c$ with some $c>0$ (see [BR], Theorem 2.5.1). By Lemma 2 we get $|F(x)| \geq c|x|^{0}$ for $|x| \gg 1$ which ends the proof in this case.

Hence we may assume throughout the rest of the proof that $K \cap(\operatorname{grad} F)^{-1}(0)$ is bounded.

Let us define

$$
\begin{aligned}
A_{i} & =\left\{x \in \mathbf{R}^{n}|| x_{k}|<| x_{i} \mid \quad \text { for } k \in\{1, \ldots, n\} \backslash\{i\}\right\}, \\
B_{i, j} & =\left\{x \in \mathbf{R}^{n}\left|x_{i}=x_{j},\right| x_{k}|\leq| x_{i} \mid \text { for } k=1, \ldots, n\right\}, \\
C_{i, j} & =\left\{x \in \mathbf{R}^{n}\left|x_{i}=-x_{j},\right| x_{k}|\leq| x_{i} \mid \text { for } k=1, \ldots, n\right\} .
\end{aligned}
$$

Since $\mathbf{R}^{n}=\bigcup A_{i} \cup \bigcup B_{i, j} \cup \cup C_{i, j}$, at least one of the sets $K \cap \bigcup A_{i}, K \cap \bigcup B_{i, j}$, $K \cap \bigcup C_{i, j}$ is unbounded. Let us consider three cases:

Case 1: $K \cap \bigcup B_{i, j}$ is unbounded. Then at least one of the sets $K \cap B_{i, j}(1 \leq i<j \leq n)$ is unbounded. Without loss of generality we can assume that this is the set $K \cap B_{n-1, n}$.

Consider the polynomial $\tilde{F}\left(X_{1}, \ldots, X_{n-1}\right)=F\left(X_{1}, \ldots, X_{n-1}, X_{n-1}\right)$ of degree $\tilde{d} \leq d$. By the inductive assumption we have $|\tilde{F}(\tilde{x})| \geq c|\tilde{x}|^{\tilde{d}-(\tilde{d}-1)^{n-1}}$ for $\tilde{x} \in \mathbf{R}^{n-1},|\tilde{x}| \gg 1$.

If we take any $x \in B_{n-1, n}, x=\left(x_{1}, \ldots, x_{n-1}, x_{n-1}\right)$ and if we set $\tilde{x}=\left(x_{1}, \ldots, x_{n-1}\right)$, then $|\tilde{x}|=|x|$ and $\tilde{F}(\tilde{x})=F(x)$. Hence $|F(x)| \geq c|x|^{\tilde{d}-(\tilde{d}-1)^{n-1}}$ for $|x| \gg 1, x \in B_{n-1, n}$. By Lemma 2 and by the inequality $\tilde{d}-(\tilde{d}-1)^{n-1} \geq d-(d-1)^{n}$ we get $|F(x)| \geq c|x|^{d-(d-1)^{n}}$ for $|x| \gg 1$.

Case 2: $K \cap \bigcup C_{i, j}$ is unbounded. The proof is analogous. 
Case 3: $K \cap \bigcup A_{i}$ is unbounded. Then at least one of the sets $K \cap A_{i}(1 \leq i \leq n)$ is unbounded. Without loss of generality we can assume that this is $K \cap A_{n}$.

Take $R>0$ large enough so that $F(x)>0$ for $|x|>R$ and let $y=\left(y_{1}, \ldots, y_{n}\right)$ be an arbitrary point in $K \cap A_{n}$ with $|y|>R$. Consider a function $f\left(x_{1}, \ldots, x_{n-1}\right)=$ $F\left(x_{1}, \ldots, x_{n-1}, y_{n}\right)$ defined for $\left|x_{i}\right|<\left|y_{n}\right|(1 \leq i<n)$. Taking into account two points, $y=\left(y_{1}, \ldots, y_{n-1}, y_{n}\right)$ and $x=\left(x_{1}, \ldots, x_{n-1}, y_{n}\right)$, where $\left|x_{i}\right|<\left|y_{n}\right|(1 \leq i<n)$, we see that $|x|=|y|$, therefore $F(x) \geq F(y)$. Hence the point $\left(y_{1}, \ldots, y_{n-1}\right)$ is a local minimum of $f$. Thus $\partial F / \partial X_{1}(y)=\ldots=\partial F / \partial X_{n-1}(y)=0$.

Summarizing, we see that for all $y \in K \cap A_{n},|y| \gg 1$ we have $\partial F / \partial X_{1}(y)=\ldots=$ $\partial F / \partial X_{n-1}(y)=0, \partial F / \partial X_{n}(y) \neq 0$. Moreover, from Lemma 1 it follows that $K \cap A_{n}$ is a one-dimensional semialgebraic manifold in a neighborhood of infinity. We want to find a parametrization of a branch at infinity of this set. To that end we employ complex algebraic geometry.

Define $H_{1}=\partial F / \partial X_{1}, \ldots, H_{n-1}=\partial F / \partial X_{n-1}$ and let $C=\left\{z \in \mathbf{C}^{n} \mid H_{1}(z)=\ldots\right.$ $\left.\ldots=H_{n-1}(z)=0\right\}$. Decompose $C$ to the union of irreducible algebraic components $C=C_{1} \cup \ldots \cup C_{s}$. Treating $\mathbf{R}^{n}$ as a subset of $\mathbf{C}^{n}$ we see that $K \cap A_{n} \cap C$ is unbounded. Hence there exists a component $C_{i}$ such that $K \cap A_{n} \cap C_{i}$ is unbounded. For simplicity put $\Gamma=C_{i}$.

We will check that $\operatorname{dim}_{\mathbf{C}} \Gamma=1$. By Lemma 1 there exists $x \in K \cap A_{n} \cap \Gamma$ for which the differentials $\mathrm{d}_{x} H_{1}, \ldots, \mathrm{d}_{x} H_{n-1}$ are linearly independent. Therefore, $\operatorname{dim}_{\mathbf{C}} \Gamma \leq$ $n-\operatorname{rank}(\Gamma, x) \leq n-\operatorname{rank}\left(\mathrm{d}_{x} H_{1}, \ldots, \mathrm{d}_{x} H_{n-1}\right)=1$ (see [BR], pages 122-135). Furthermore, $\Gamma$ is unbounded, so $\operatorname{dim}_{\mathbf{C}} \Gamma=1 .\left({ }^{1}\right)$

Next, we will check that $\operatorname{deg} \Gamma \leq(d-1)^{n-1}$. Let us recall an invariant $\delta$ of algebraic sets introduced in Łojasiewicz's book ([Ło] pages 419-420): Let $W=W_{1} \cup \ldots \cup W_{s}$ be a decomposition of an algebraic set $W$ to irreducible components. Then, by definition $\delta(W)=\sum_{i=1}^{s} \operatorname{deg} W_{i}$. We will use the inequality $\delta(W \cap V) \leq \delta(W) \delta(V)$. Applying this property to the set $C$ we see that $\operatorname{deg} \Gamma \leq \delta(C)=\delta\left(\left\{H_{1}=0\right\} \cap \ldots \cap\left\{H_{n-1}=0\right\}\right) \leq$ $\prod_{i=1}^{n-1} \delta\left(\left\{H_{i}=0\right\}\right) \leq(d-1)^{n-1}$.

Further, we will consider $\mathbf{C}^{n}$ as a affine part of the projective space $\mathbf{C} P^{n}$. We will use the natural identification between $\left(x_{1}, \ldots, x_{n}\right) \in \mathbf{C}^{n}$ and $\left[1, x_{1}, \ldots, x_{n}\right] \in \mathbf{C} P^{n}$. With the use of this identification we can treat $K, A_{n}$ and $\Gamma$ as subsets of $\mathbf{C} P^{n}$.

Since $K \cap A_{n} \cap \Gamma$ is an unbounded set and $\mathbf{C} P^{n}$ is compact, there exists a point $a$ in the hyperplane at infinity $\left\{\left[x_{0}, \ldots, x_{n}\right] \in \mathbf{C} P^{n} \mid x_{0}=0\right\}$ such that $a \in \operatorname{cl}\left(K \cap A_{n} \cap \Gamma\right)$.

The homogeneous coordinates of $a$ can be chosen such that $a=\left[0, a_{1}, \ldots, a_{n-1}, 1\right]$. Indeed, for all $x \in A_{n}$ we have $\left|x_{i}\right|<\left|x_{n}\right|$ for $1 \leq i<n$. Since $a \in \operatorname{cl}\left(A_{n}\right),\left|a_{i}\right| \leq\left|a_{n}\right|$ for $1 \leq i<n$. Therefore, the last coordinate $a_{n}$ does not vanish and by homogeneity we can assume that $a_{n}=1$.

Let $\bar{\Gamma}$ be the projective closure of the curve $\Gamma$. Since $a \in \bar{\Gamma}$, according to [Ło] (pages 173-176) there exists a finite sequence of injective holomorphic parametrizations $\gamma_{i}:(D, 0) \rightarrow(\bar{\Gamma}, a)(1 \leq i \leq l)$, where $D=\{t \in \mathbf{C}|| t \mid<\delta\}$, such that the curve $\bar{\Gamma}$ is the union $\gamma_{1}(D) \cup \ldots \cup \gamma_{l}(D)$ in some neighborhood of $a$. These parametrizations are of the form

$$
\gamma_{i}(t)=\left[t^{d_{i}}, \gamma_{i, 1}(t), \ldots, \gamma_{i, n-1}(t), 1\right]
$$

Furthermore, we can assume that the real branches of $\bar{\Gamma}$ are parametrized such that $\left(t^{d_{i}}, \gamma_{i, 1}(t), \ldots, \gamma_{i, n-1}(t)\right) \in \mathbf{R}^{n}$ if and only if $t \in \mathbf{R}$. This can be done by substituting

$\left.{ }^{1}\right)$ If $\operatorname{dim}_{\mathbf{C}} \Gamma=0$, then $\Gamma$ would consist of one point. 
$\gamma_{i}\left(\xi_{i} t\right)$, where $\xi_{i}$ is an appropriate $d_{i}$-th root of unity and by shrinking $\delta$ if necessary (see $[\mathrm{Mi}]$ or $[\mathrm{Du}]$ for the details).

Let $H=H\left(X_{0}, \ldots, X_{n}\right)$ be the homogenization of the polynomial $\partial F / \partial X_{n}$. Recall that it means that $H$ is a homogeneous polynomial of degree $\operatorname{deg} H=\operatorname{deg} \partial F / \partial X_{n}$ such that $H\left(1, X_{1}, \ldots, X_{n}\right)=\partial F / \partial X_{n}\left(X_{1}, \ldots, X_{n}\right)$. We can calculate the intersection multiplicity of the curve $\bar{\Gamma}$ and the hypersurface $\{H=0\}$ at $a$ using the formula

$$
\iota_{a}(\bar{\Gamma},\{H=0\})=\sum_{i=1}^{l} \operatorname{ord}_{0}\left(H \circ \gamma_{i}\right)
$$

(see [Sh], pages 190-194). By Bézout's theorem $\iota_{a}(\bar{\Gamma},\{H=0\}) \leq(\operatorname{deg} \bar{\Gamma})(\operatorname{deg} H) \leq$ $(d-1)^{n}$. Hence $\operatorname{ord}_{0}\left(H \circ \gamma_{i}\right) \leq(d-1)^{n}$ for $i=1, \ldots, l$.

One has $a \in \operatorname{cl}\left(K \cap A_{n} \cap \Gamma\right)$. Hence there exists $i(1 \leq i \leq l)$ such that $a \in \operatorname{cl}(K \cap$ $\left.A_{n} \cap \gamma_{i}(D)\right)$. Since $\gamma_{i}$ is a proper map, $0 \in \operatorname{cl}\left(\gamma^{-1}\left(K \cap A_{n}\right)\right)$. Furthermore, we see by the definition of $\gamma_{i}$ that $\gamma^{-1}\left(K \cap A_{n}\right)$ is a semianalytic subset of $\mathbf{R}$. Therefore there exists $\epsilon>0$ such that $\gamma_{i}((0, \epsilon)) \subset K \cap A_{n}$ or $\gamma_{i}((-\epsilon, 0)) \subset K \cap A_{n}$ (see [BM] for the definition and basic properties of semianalytic sets). In the rest of the proof we assume the former case (the proof for the case $\gamma_{i}((-\epsilon, 0)) \subset K \cap A_{n}$ is similar). We will again treat $K, A_{n}$ and $\Gamma$ as subsets of $\mathbf{C}^{n}$.

Set the following meromorphic map

$$
\phi:\{t \in \mathbf{C}|0<| t \mid<\epsilon\} \ni t \rightarrow\left(\gamma_{i, 1}(t) / t^{d_{i}}, \ldots, \gamma_{i, n-1}(t) / t^{d_{i}}, 1 / t^{d_{i}}\right) \in \mathbf{C}^{n} .
$$

Notice that $\phi(\{t \in \mathbf{C}|0<| t \mid<\epsilon\}) \subset \Gamma$ and that $\phi((0, \epsilon))$ is an unbounded semialgebraic subset of $K \cap A_{n}$.

We estimate the order of $F \circ \phi$ at zero. Either $\operatorname{ord}_{0}(F \circ \phi)=0$ or by the equation

$$
(F \circ \phi)^{\prime}=\left(\frac{\partial F}{\partial X_{1}} \circ \phi\right) \phi_{1}^{\prime}+\ldots+\left(\frac{\partial F}{\partial X_{n}} \circ \phi\right) \phi_{n}^{\prime}=\left(\frac{\partial F}{\partial X_{n}} \circ \phi\right) \phi_{n}^{\prime}
$$

we have $\operatorname{ord}_{0}(F \circ \phi)=\operatorname{ord}_{0}\left(\partial F / \partial X_{n} \circ \phi\right)-d_{i}$.

On the other hand we have

$$
\begin{aligned}
\frac{\partial F}{\partial X_{n}}(\phi(t)) & =H\left(1, \gamma_{i, 1}(t) / t^{d_{i}}, \ldots, \gamma_{i, n-1}(t) / t^{d_{i}}, 1 / t^{d_{i}}\right) \\
& =t^{-d_{i} \operatorname{deg} H} H\left(t^{t_{i}}, \gamma_{i, 1}(t), \ldots, \gamma_{i, n-1}(t), 1\right)=t^{-d_{i} \operatorname{deg} H} H\left(\gamma_{i}(t)\right) .
\end{aligned}
$$

Since deg $H=d-1$ and $\operatorname{ord}_{0}\left(H \circ \gamma_{i}\right) \leq(d-1)^{n}$, we conclude that $\operatorname{ord}_{0}\left(\partial F / \partial X_{n} \circ \phi\right) \leq$ $(d-1)^{n}-d_{i}(d-1)$. By this inequality and the preceding equalities we have $\operatorname{ord}_{0}(F \circ \phi) \leq$ $(d-1)^{n}-d_{i} d$ or $\operatorname{ord}_{0}(F \circ \phi)=0$.

Rem ark. If $f, g:\{t \in \mathbf{C}|0<| t \mid<\epsilon\} \rightarrow \mathbf{C}, f \neq 0, g \neq 0$ are meromorphic functions, then there exist constants $c, \epsilon_{1}>0$ such that $|f(t)| \geq c|g(t)|^{\operatorname{ord}_{0} f / \operatorname{ord}_{0} g}$ for all $t \in \mathbf{C}, 0<|t|<\epsilon_{1}$.

The proof of this fact is simple and is left to the reader. By the remark and by the fact that $\phi((0, \epsilon)) \subset A_{n}$ implies $|\phi(t)|=\left|\phi_{n}(t)\right|$ for $t \in(0, \epsilon)$, we obtain an inequality

$$
|F(\phi(t))| \geq c|\phi(t)|^{\operatorname{ord}_{0}(F \circ \phi) / \operatorname{ord}_{0}\left(\phi_{n}\right)} \quad \text { for } \quad t \in\left(0, \epsilon_{1}\right)
$$

with some positive constants $c, \epsilon_{1}$. By Lemma 2 we have

$$
|F(x)| \geq c|x|^{\operatorname{ord}_{0}(F \circ \phi) / \operatorname{ord}_{0}\left(\phi_{n}\right)} \quad \text { for } \quad|x| \gg 1 .
$$


Moreover, the exponent $\operatorname{ord}_{0}(F \circ \phi) / \operatorname{ord}_{0}\left(\phi_{n}\right) \geq\left((d-1)^{n}-d_{i} d\right) /\left(-d_{i}\right)=d-(d-1)^{n} / d_{i} \geq$ $d-(d-1)^{n}$ or is equal zero and thus

$$
|F(x)| \geq c|x|^{d-(d-1)^{n}} \quad \text { for } \quad|x| \gg 1 .
$$

4. Concluding remarks. In the course of the proof we have found a parametrization $\phi$ of the set $K$ at infinity such that $|F(x)| \geq c|x|^{\operatorname{ord}_{0}(F \circ \phi) / \operatorname{ord}_{0}\left(\phi_{n}\right)}$ for $|x| \gg 1$. By a slight modification of the proof one can show that the number $\operatorname{ord}_{0}(F \circ \phi) / \operatorname{ord}_{0}\left(\phi_{n}\right)$ is the Łojasiewicz exponent at infinity for the polynomial $F$, i.e. the largest exponent $\alpha$ for which the estimate $|F(x)| \geq$ const. $|x|^{\alpha}$ is true for $|x| \gg 1$.

We have checked that $\operatorname{ord}_{0}(F \circ \phi) \leq(d-1)^{n}-d \operatorname{ord}_{0}\left(\phi_{n}\right)$ or $\operatorname{ord}_{0}(F \circ \phi)=0$. One can also prove the inequality $(d-1)^{n-1} \leq \operatorname{ord}_{0}\left(\phi_{n}\right)<0$. As a result, there is only a finite number of fractions which can be the Lojasiewicz exponents for polynomials of fixed number of variables $n$ and of fixed degree $d$.

So far I have not found a polynomial $F$ for which the Lojasiewicz exponent $L_{\infty}(F)=$ $d-(d-1)^{n}$. For example for the polynomial $F\left(X_{1}, \ldots, X_{n}\right)=\left(X_{2} X_{1}^{m-1}-1\right)^{2}+$ $\left(X_{3}-X_{2}^{m}\right)^{2}+\ldots+\left(X_{n}-X_{n-1}^{m}\right)^{2}+X_{n}^{2 m}$ of degree $d=2 m$ we have $L_{\infty}(F)=d-\left(1 / 2^{n-1}\right) d^{n}$. This suggests that Theorem 1 could be essentially sharpened.

I want to express my thanks to Stanisław Spodzieja for pointing out a mistake in the first version of this paper.

\section{References}

[BR] B. Benedetti, J. J. Risler, Real Algebraic and Semi-algebraic Sets, Hermann, Paris, 1990.

[BM] E. Bierstone, P. D. Milman, Semianalytic and subanalytic sets, Inst. Hautes Études Sci. Publ. Math. 67 (1988), 5-42.

[Du] Z. Duszak, Indeks rzeczywistych odwzorowań analitycznych, Doctoral Thesis, Uniwersytet Łódzki, 1987.

[Ko] J. Kollár, Sharp effective Nullstellensatz, J. Amer. Math. Soc. 1 (1988), 963-975.

[Ło] S. Łojasiewicz, Introduction to Complex Algebraic Geometry, Birkhäuser, Basel, 1991.

[Mi] J. W. Milnor, Singular Points of Complex Hypersurfaces, Princeton Univ. Press, Princeton, 1968.

[Sh] I. R. Shafarevich, Basic Algebraic Geometry, Springer, New York, 1974. 